\title{
Rates and costs of invasive pneumococcal disease and pneumonia in persons with underlying medical conditions
}

\author{
Derek Weycker ${ }^{1 *}$, Raymond A. Farkouh², David R. Strutton², John Edelsberg ${ }^{1}$, Kimberly M. Shea ${ }^{3}$
} and Stephen I. Pelton ${ }^{3,4}$

\begin{abstract}
Background: The presence of certain underlying medical conditions is known to increase the risk of pneumococcal disease in persons of all ages and across a wide spectrum of conditions, as demonstrated in two recent evaluations. Corresponding estimates of attributable economic costs have not been well characterized. We thus undertook a retrospective evaluation to estimate rates and costs of pneumococcal disease among children and adults with and without underlying medical conditions in the United States.

Methods: Data were obtained from three independent healthcare claims repositories. The study population included all persons enrolled in participating health plans during 2007-2010, and was stratified into subgroups based on age and risk profile: healthy; at-risk, due to selected comorbid conditions; and high-risk, due to selected immunocompromising conditions. At-risk and high-risk conditions, as well as episodes of invasive pneumococcal disease (IPD) and all-cause pneumonia (PNE), were identified via diagnosis, procedure, and drug codes. Rates and healthcare costs of IPD and PNE (2010US\$) among at-risk and high-risk persons were compared with those from age-stratified healthy counterparts using incidence rate ratios (IRR) and cost ratios.

Results: Rates of IPD and PNE were consistently higher among at-risk persons (IRR $=4.1[95 \% \mathrm{Cl} 3.9-4.3]$ and 4.5 [4.49-4.53]) and high-risk persons (IRR $=10.3$ [9.7-11.0] and 8.2 [8.2-8.3]) of all ages versus their healthy counterparts. Rates were notably high for at-risk persons with $\geq 2$ conditions (IRR $=9.0$ [8.4-9.7] and 10.3 [10.3-10.4]), as well as those with asthma (IRR $=3.4$ [3.0-3.8] and 4.5 [4.47-4.53]) or diabetes (IRR $=4.3$ [4.0-4.6] and 4.7 [4.6-4.7]). Healthcare costs totaled $\$ 21.7$ million per 100,000 at-risk person-years and $\$ 58.5$ million per 100,000 high-risk person-years, which were 8.7 [8.5-8.8] and 23.4 [22.9-23.8] times higher than corresponding costs for healthy persons.

Conclusions: Rates and costs of IPD and PNE are substantially higher among persons with certain chronic and immunocompromising conditions versus those without any such conditions. Rates and costs for persons with asthma and diabetes were especially increased, and rates and costs for individuals with $\geq 2$ at-risk conditions approached those among persons with high-risk conditions.
\end{abstract}

Keywords: Streptococcus pneumoniae, Pneumococcal infections, Pneumonia, Comorbidity, Cost and cost analysis

\section{Background}

Streptococcus pneumoniae (pneumococcus) has long been recognized as a major cause of serious but uncommon invasive diseases such as meningitis and bacteremic pneumonia, and much more common non-invasive diseases such as non-bacteremic pneumonia and acute

\footnotetext{
* Correspondence: dweycker@pai2.com

${ }^{1}$ Policy Analysis Inc. (PAI), Four Davis Court, Brookline, MA 02445, USA Full list of author information is available at the end of the article
}

otitis media. Not surprisingly then, the economic burden of pneumococcal disease in the United States (US) has been reported to be substantial. In one recent evaluation, Huang and colleagues estimated the direct cost of pneumococcal disease among persons of all ages in the US to have been $\$ 3.5$ billion in 2004 [1]. In another evaluation, Weycker and colleagues estimated the direct cost of pneumococcal disease among older US adults (i.e., persons aged $\geq 50$ years) to have been $\$ 3.7$ billion in 
2007 [2]. Finally, Wroe and colleagues projected that from 2004-2040, as the size of the US population grows (especially the elderly) and absent intervention, the number of hospitalizations for pneumococcal pneumonia will nearly double and the total economic burden of pneumococcal pneumonia will increase by $\$ 2.5$ billion annually [3].

In the study by Weycker et al., the effect of patients' underlying risk of pneumococcal disease on both the cost per case of disease and the overall economic burden of pneumococcal disease were examined, but only to a limited extent [2]. We therefore examined in greater detail, and for persons of all ages, the clinical and economic burden of pneumococcal disease in the US in relation to underlying risk status. Risk profiles were determined by the presence of selected underlying chronic illnesses that are currently listed in guidelines promulgated by the US Advisory Committee on Immunization Practices (ACIP) as indications for pneumococcal vaccination, as well as conditions that might increase the risk of infection based on limited data from other studies [4-7].

\section{Methods}

\section{Study design}

A retrospective cohort design was employed. Study cohorts were identified at the beginning of each calendar year of observation-from 2007 to 2010-and study subjects were stratified in terms of age and the presence of underlying medical conditions (i.e., risk profile) based on information recorded at any time prior to January $1^{\text {st }}$ of that calendar year. For each cohort, episodes of invasive pneumococcal disease (IPD) and all-cause pneumonia (PNE) were ascertained during the 1-year period beginning on January $1^{\text {st }}$ of each corresponding year and ending on December $31^{\text {st }}$ of that year (or date of loss to followup, if earlier). (All-cause PNE was selected as a study measure-rather than pneumococcal pneumonia-because pneumococcal pneumonia is under-reported in healthcare claims and $\sim 30 \%$ of all cause PNE is estimated to be due to Streptococcus pneumoniae [7, 8]. Stratum-specific rates of IPD and PNE (per 100,000 person-years) were estimated over the entire study period. Subjects who met criteria for inclusion in multiple calendar years contributed data to each cohort for which they were eligible.

\section{Data source}

Data were obtained from three large integrated healthcare claims repositories and spanned January 1, 2006 through December 31, 2010. The three databasesTruven Health Analytics MarketScan ${ }^{\circ}$ Commercial Claims and Encounters and Medicare Supplemental and Coordination of Benefits Databases, Intercontinental Marketing Services' (IMS) LifeLink ${ }^{\text {mi }}$ PharMetrics Health
Plan Claims Database, and OPTUM Research Databaseinclude medical (i.e., facility and professional-service) claims and outpatient pharmacy claims from private US health plans. Together, these three geographically diverse repositories capture healthcare claims information for $>35$ million plan members annually.

Data available from each facility and professionalservice claim included dates and places of service, diagnoses (International Classification of Diseases, Ninth Revision, Clinical Modification [ICD-9-CM]), procedures performed/services rendered [ICD-9-CM, Healthcare Common Procedure Coding System (HCPCS)], and quantity of services (professional-service claims). Data available for each outpatient pharmacy claim included the drug dispensed, dispensing date, quantity dispensed, and number of days supplied. Medical and pharmacy claims also included amounts paid by health plans and patients for services rendered, or standardized estimates of the cost of services. In addition, selected demographic and eligibility information (including age/year of birth, sex, geographic region of residence, and dates of plan eligibility) were available.

The study databases were de-identified prior to their release to study investigators. The study databases have been evaluated and certified by independent third parties to be in compliance with the Health Insurance Portability and Accountability Act (HIPAA) of 1996 statistical de-identification standards and to satisfy the conditions set forth in Sections 164.514 (a)-(b)1ii of the HIPAA Privacy Rule regarding the determination and documentation of statistically de-identified data. Use of the study databases for health services research is therefore fully compliant with the HIPAA Privacy Rule and federal guidance on Public Welfare and the Protection of Human Subjects [9]. Permission to use the data sources for analyses described herein was requested by study investigators and was granted by the data vendors. Detailed descriptions of the three healthcare claims repositories are provided in the Additional file 1.

\section{Study population}

The study population comprised all persons of all ages who were enrolled in participating health plans on the first day of one or more calendar years from 2007 to 2010. Study subjects were stratified based on their age (0-17, 18-64, and $\geq 65$ years) and risk profile ("healthy", "at-risk", and "high-risk") as of the beginning of each year. At-risk persons included those who were immunocompetent with $\geq 1$ chronic medical condition. High-risk persons included those who were immunocompromised, immunosuppressed, or had a cochlear implant. At-risk and high-risk categories were mutually exclusive and thus, for example, persons considered immunosuppressed because of cancer were included in the high-risk 
category only, even if they also had an at-risk condition. Healthy persons included those without evidence of atrisk or high-risk conditions.

The lists of at-risk and high-risk conditions included all those set forth by the ACIP in its recommendations for pneumococcal vaccination; the at-risk list also included the following conditions: asthma, chronic steroid use, trisomy 21, prematurity/low birth weight, neuromuscular/seizures disorders, rheumatoid arthritis, systemic lupus erythematosis, and Crohn's disease [10]. At-risk and high-risk conditions were ascertained using information (i.e., ICD-9-CM diagnosis codes, ICD-9-CM/ HCPCS procedure codes, and drug codes from the HCPCS/National Drug Code [NDC] systems) recorded any time prior to the beginning of the study year. Persons with at-risk conditions were stratified by the number of conditions; those with asthma and diabetes were further stratified by disease severity. Operational algorithms that were employed to identify at-risk and highrisk conditions are set forth in Additional file 1: Tables S1 and S2.

Persons who were not continuously eligible for comprehensive health (i.e., medical and drug) benefits for at least 1 year prior to January $1^{\text {st }}$ of $\geq 1$ corresponding years were excluded from the study population. Infants (i.e., those $<12$ months of age as of January $1^{\text {st }}$ ) in a given year, were not subject to this exclusionary criterion.

\section{Study measures}

Study measures included episodes and associated healthcare costs of selected manifestations of pneumococcal infection, including IPD and PNE. All-cause pneumonia was chosen as an outcome measure in preference to pneumococcal pneumonia because, although S. pneumoniae infection is recognized as the most common cause of pneumonia, the diagnostic code for pneumococcal pneumonia appears uncommonly in healthcare claims data (due to the relatively small number of times $S$. pneumoniae is successfully cultured in clinical practice from patients with pneumonia).

Episodes of IPD and PNE were ascertained beginning on January $1^{\text {st }}$ and ending on December $31^{\text {st }}$ of each study year (or the date of loss to follow-up, if prior to December 31st). Disease episodes requiring inpatient care were identified using operational algorithms based on ICD-9-CM diagnosis codes, and spanned the duration of hospitalization. Episodes of IPD requiring outpatient care only were identified based on ICD-9-CM diagnosis codes and HCPCS/NDC codes for antibiotic therapy ( \pm 5 days), while episodes of PNE requiring outpatient care only were identified based on ICD-9-CM diagnosis codes, CPT codes for chest $\mathrm{x}$-ray, and HCPCS/ NDC codes for antibiotic therapy ( \pm 5 days); outpatient episodes spanned a maximum of 90 days (Additional file 1 :
Table S3). Multiple episodes during a given study year were included if they were separated by at least 90 days.

A disease-attributable approach was used to estimate healthcare costs whereby expenditures for diseaserelated services rendered during the episode of care were tallied. Disease-related services were identified based on diagnosis codes (principal diagnosis only on inpatient claims), procedure codes, and drug codes. The cost of inpatient episodes included hospitalizations, ambulatory visits, and pharmacotherapy during the episode; the cost of outpatient episodes included all outpatient visits and pharmacotherapy during the episode. Costs were estimated using healthcare expenditures reported on claims (i.e., amounts reimbursed to healthcare providers by health plans and patients for services rendered), and were expressed in 2010 US dollars.

\section{Analyses}

Crude analyses were undertaken to estimate rates of disease episodes (per 100,000 person-years) within each age group by risk profile and individual risk condition, using data from all three study repositories. Differences in rates of disease-and corresponding $95 \%$ confidence intervals (CIs)-between at-risk/high-risk persons and healthy counterparts were expressed as incidence rate ratios (IRR) using Poisson regression analysis.

Mean episodic costs of IPD and PNE by age and risk profile were estimated using data only from the MarketScan Database since it was the only source for which we had access to detailed patient-level data. Costs of IPD and PNE (per 100,000 person-years) by age and risk profile-as well as corresponding differences and $95 \%$ CIs between risk groups-were estimated by combining sampled rates of disease (from all three data sources) and sampled age- and risk-specific unit costs (from MarketScan) via techniques of non-parametric bootstrapping (percentile method, 1000 replications with replacement). All analyses were conducted using $\mathrm{SAS}^{\circ} 9.3$ for Windows ${ }^{\oplus}$ (SAS Institute Inc., Cary, NC, USA).

\section{Results}

\section{Risk profiles}

Children (aged 0-17 years) who qualified for inclusion in the study contributed a total of 26.5 million personyears of observation (Table 1). Approximately $92 \%$ of children had none of the selected chronic or immunocompromising conditions, $8 \%$ had $\geq 1$ at-risk condition (and no high-risk conditions), and $<1 \%$ had a high-risk condition. Among children with at-risk conditions, asthma (63\%), prematurity/low birth weight (13\%), neuromuscular/seizure disorders (10\%), and chronic lung disease $(10 \%)$ were the most common. Of children with $\geq 1$ at-risk condition, $7 \%$ had $\geq 2$ such conditions. 
Adults aged 18-64 years and $\geq 65$ years contributed 79.9 million and 11.4 million person-years of observation, respectively. Approximately $79 \%$ of adults aged 18-64 years had none of the selected chronic or immunocompromising conditions, while $17 \%$ had $\geq 1$ at-risk condition (and no high-risk conditions) and $4 \%$ had a high-risk condition. The most common conditions in this age group were diabetes (43\%), chronic heart disease (23\%), and asthma (16\%); $18 \%$ had $\geq 2$ of any of the listed at-risk conditions. The prevalence of at-risk and high-risk conditions in persons aged $\geq 65$ years was $39 \%$ and $15 \%$, respectively; the most common conditions were chronic heart disease (52 \%), diabetes (50\%), and chronic lung disease (19\%); $32 \%$ had $\geq 2$ such conditions.

\section{Clinical and economic burden}

Rates of IPD and PNE were consistently higher among at-risk persons of all ages (IRR $=4.1$ [95 \% CI 3.9-4.3] and 4.5 [4.49-4.53]) and high-risk persons of all ages $(\mathrm{IRR}=10.3$ [9.7-11.0] and $8.2[8.2-8.3])$ versus their healthy counterparts. Rates were notably high for at-risk persons with $\geq 2$ at-risk conditions (IRR $=9.0$ [8.4-9.7] and $10.3[10.3-10.4]$ ), as well as those with asthma (IRR $=3.4$ [3.0-3.8] and 4.5 [4.47-4.53]) or diabetes (IRR $=4.3$ [4.0-4.6] and 4.7 [4.6-4.7]).

Cost per IPD episode was generally similar across risk groups, while the cost per PNE episode was highest for the high-risk population followed by the at-risk and healthy populations (Table 2). Differences in economic costs of IPD and PNE per 100,000 person-years, by risk profile and number of at-risk conditions, were roughly the same as those for disease rates (Figs. 1 and 2).

Although high-risk persons were a small proportion of the total, they accounted for a substantial proportion of total costs (Fig. 3). On an overall basis, high-risk persons constituted only about $4 \%$ of the population, but accounted for $18 \%$ of cases of IPD/PNE and $30 \%$ of the total cost of IPD/PNE. Similarly, persons with $\geq 2$ at risk conditions, while constituting only $3 \%$ of the total population, accounted for $21 \%$ of total IPD/PNE costs. Economic costs increased in a graded fashion by severity

Table 1 Rates of invasive pneumococcal disease and all-cause pneumonia among healthy, at risk, and high-risk persons

\begin{tabular}{|c|c|c|c|c|c|c|c|c|c|}
\hline & \multirow{2}{*}{\multicolumn{3}{|c|}{ No. of person-years }} & \multicolumn{6}{|c|}{ Invasive Pneumococcal Disease } \\
\hline & & & & \multicolumn{2}{|c|}{ Age $<18$ years } & \multicolumn{2}{|c|}{ Age $18-64$ years } & \multicolumn{2}{|c|}{ Age $\geq 65$ years } \\
\hline & $\begin{array}{l}\text { Age } \\
<18 \text { years }\end{array}$ & $\begin{array}{l}\text { Age } \\
\text { 18-64 years }\end{array}$ & $\begin{array}{l}\text { Age } \\
\geq 65 \text { years }\end{array}$ & $\begin{array}{l}\text { Rate per } \\
100 \mathrm{~K}\end{array}$ & $\begin{array}{l}\text { Rate ratios } \\
(95 \% \mathrm{Cl})\end{array}$ & $\begin{array}{l}\text { Rate per } \\
100 \mathrm{~K}\end{array}$ & $\begin{array}{l}\text { Rate ratios } \\
(95 \% \mathrm{Cl}) \\
\end{array}$ & $\begin{array}{l}\text { Rate per } \\
100 \mathrm{~K}\end{array}$ & $\begin{array}{l}\text { Rate ratios } \\
(95 \% \mathrm{Cl})\end{array}$ \\
\hline \multicolumn{10}{|l|}{ Risk Group } \\
\hline Healthy & $24,406,759$ & $63,445,448$ & $5,389,930$ & 2.5 & - & 2.7 & - & 8.3 & - \\
\hline At-Risk & $2,002,374$ & $13,368,935$ & $4,579,505$ & 5.9 & $2.4(2.0-2.9)$ & 9.3 & $3.4(3.2-3.7)$ & 23.0 & $2.8(2.5-3.1)$ \\
\hline Alcoholism & 4591 & 333,634 & 23,905 & 0.0 & - & 20.4 & $7.5(5.9-9.6)$ & 41.8 & $5.0(2.7-9.4)$ \\
\hline Asthma & $1,252,480$ & $2,185,510$ & 362,183 & 3.7 & $1.5(1.1-2.0)$ & 9.6 & $3.6(3.1-4.1)$ & 34.2 & $4.1(3.4-5.0)$ \\
\hline Chronic heart disease & 136,206 & $3,082,998$ & $2,363,798$ & 13.9 & $5.7(3.6-8.9)$ & 11.5 & $4.3(3.8-4.8)$ & 26.6 & $3.2(2.8-3.6)$ \\
\hline Chronic liver disease & 3759 & 292,697 & 50,540 & 26.6 & $10.8(1.5-76.7)$ & 24.6 & $9.1(7.2-11.5)$ & 53.4 & $6.4(4.4-9.5)$ \\
\hline Chronic lung disease & 192,731 & $1,251,143$ & 882,061 & 8.3 & $3.4(2.0-5.5)$ & 27.0 & $10.0(8.9-11.2)$ & 51.1 & $6.2(5.4-7.0)$ \\
\hline Chronic use of oral steroids & 22,508 & 275,267 & 65,775 & 13.3 & $5.4(1.7-16.8)$ & 8.0 & $3.0(1.9-4.5)$ & 15.2 & $1.8(1.0-3.4)$ \\
\hline Diabetes & 88,848 & $5,721,158$ & $2,267,133$ & 5.6 & $2.3(0.9-5.5)$ & 9.5 & $3.5(3.2-3.9)$ & 21.1 & $2.5(2.2-2.9)$ \\
\hline Down's syndrome & 20,657 & 7649 & 63 & 19.4 & $7.9(2.9-21.0)$ & 13.1 & $4.8(0.7-34.4)$ & 0.0 & - \\
\hline Neuromuscular/seizure disorders & 193,657 & 507,134 & 104,864 & 11.9 & $4.8(3.2-7.3)$ & 12.8 & $4.7(3.7-6.1)$ & 38.1 & $4.6(3.3-6.3)$ \\
\hline Short gestation/low birthweight & 249,576 & 34,580 & 90 & 12.0 & $4.9(3.4-7.0)$ & 5.8 & $2.1(0.5-8.6)$ & 0.0 & - \\
\hline Rheumatoid arthritis/Crohn's/Lupus & - & 579,373 & 162,206 & - & - & 17.8 & $6.6(5.4-8.0)$ & 33.3 & $4.0(3.0-5.3)$ \\
\hline Smokers & 5019 & $2,128,945$ & 180,504 & 0.0 & - & 12.5 & $4.6(4.1-5.3)$ & 34.9 & $4.2(3.2-5.5)$ \\
\hline High-Risk & 119,692 & $3,062,400$ & $1,774,181$ & 48.5 & $19.6(15.0-25.7)$ & 26.3 & $9.7(9.0-10.6)$ & 36.7 & $4.4(3.9-5.0)$ \\
\hline Chronic renal failure & 17,112 & 356,088 & 344,160 & 64.3 & $26.1(14.4-47.3)$ & 47.2 & $17.5(14.9-20.5)$ & 50.0 & $6.0(5.0-7.2)$ \\
\hline Cochlear implant & 1989 & 2517 & 1144 & 50.3 & $20.4(2.9-145.0)$ & 0.0 & - & 87.4 & $10.5(1.5-74.8)$ \\
\hline Congenital immunodeficiency & 21,047 & 76,771 & 14,392 & 42.8 & $17.3(9.0-33.5)$ & 87.3 & $32.3(25.3-41.2)$ & 118.1 & $14.2(8.8-23.1)$ \\
\hline Diseases of white blood cells & 15,493 & 149,802 & 46,869 & 122.6 & $49.7(31.5-78.5)$ & 62.1 & $23.0(18.6-28.3)$ & 110.9 & $13.3(10.0-17.8)$ \\
\hline Functional/anatomic asplenia & 19,321 & 109,298 & 42,976 & 119.0 & $48.3(31.8-73.2)$ & 93.3 & $34.5(28.3-42.2)$ & 116.3 & $14.0(10.5-18.7)$ \\
\hline HIV & 1484 & 193,184 & 7306 & 0.0 & - & 46.6 & $17.2(13.9-21.3)$ & 27.4 & $3.3(0.8-13.2)$ \\
\hline Immuno. conditions/drugs & 63,702 & $2,495,776$ & $1,523,021$ & 54.9 & $22.3(15.8-31.3)$ & 24.4 & $9.0(8.2-9.9)$ & 36.4 & $4.4(3.9-5.0)$ \\
\hline
\end{tabular}


Table 1 Rates of invasive pneumococcal disease and all-cause pneumonia among healthy, at risk, and high-risk persons (Continued)

\begin{tabular}{|c|c|c|c|c|c|c|}
\hline & \multicolumn{6}{|c|}{ All cause pneumonia } \\
\hline & \multicolumn{2}{|l|}{ Age $<18$ years } & \multicolumn{2}{|c|}{ Age 18-64 years } & \multicolumn{2}{|l|}{ Age $\geq 65$ years } \\
\hline & Rate per $100 \mathrm{~K}$ & Rate ratios ${ }^{\mathrm{a}}(95 \% \mathrm{Cl})$ & Rate per $100 \mathrm{~K}$ & Rate ratios ${ }^{\mathrm{a}}(95 \% \mathrm{Cl})$ & Rate per $100 \mathrm{~K}$ & Rate ratios ${ }^{\mathrm{a}}(95 \% \mathrm{Cl})$ \\
\hline \multicolumn{7}{|l|}{ Risk Group } \\
\hline Healthy & 587 & - & 458 & - & 1874 & - \\
\hline At-Risk & 1801 & $3.1(3.0-3.1)$ & 1652 & $3.6(3.6-3.6)$ & 5662 & $3.0(3.0-3.0)$ \\
\hline Alcoholism & 501 & $0.9(0.6-1.3)$ & 2109 & $4.6(4.5-4.7)$ & 7400 & $3.9(3.8-4.1)$ \\
\hline Asthma & 1718 & $2.9(2.9-3.0)$ & 2078 & $4.5(4.5-4.6)$ & 8570 & $4.6(4.5-4.6)$ \\
\hline Chronic heart disease & 3699 & $6.3(6.1-6.5)$ & 2533 & $5.5(5.5-5.6)$ & 7100 & $3.8(3.8-3.8)$ \\
\hline Chronic liver disease & 3910 & $6.7(5.7-7.8)$ & 3002 & $6.6(6.4-6.7)$ & 7742 & $4.1(4.0-4.3)$ \\
\hline Chronic lung disease & 3488 & $5.9(5.8-6.1)$ & 4802 & $10.5(10.4-10.6)$ & 12,379 & $6.6(6.6-6.7)$ \\
\hline Chronic use of oral steroids & 1080 & $1.8(1.6-2.1)$ & 1164 & $2.5(2.5-2.6)$ & 3696 & $2.0(1.9-2.1)$ \\
\hline Diabetes & 808 & $1.4(1.3-1.5)$ & 1683 & $3.7(3.6-3.7)$ & 5266 & $2.8(2.8-2.8)$ \\
\hline Down's syndrome & 5514 & $9.4(8.9-10.0)$ & 2915 & $6.4(5.6-7.3)$ & 6316 & $3.4(1.3-9.0)$ \\
\hline Neuromuscular/seizure disorders & 2536 & $4.3(4.2-4.4)$ & 2254 & $4.9(4.8-5.0)$ & 8539 & $4.6(4.5-4.7)$ \\
\hline Short gestation/low birthweight & 2768 & $4.7(4.6-4.8)$ & 581 & $1.3(1.1-1.5)$ & 8864 & $4.7(2.4-9.5)$ \\
\hline Rheumatoid arthritis/Crohn's/Lupus & - & - & 2131 & $4.7(4.6-4.7)$ & 6465 & $3.5(3.4-3.5)$ \\
\hline Smokers & 777 & $1.3(1.0-1.8)$ & 1858 & $4.1(4.0-4.1)$ & 6691 & $3.6(3.5-3.6)$ \\
\hline High-Risk & 3757 & $6.4(6.2-6.6)$ & 3094 & $6.8(6.7-6.8)$ & 7594 & $4.1(4.0-4.1)$ \\
\hline Chronic renal failure & 4202 & $7.2(6.7-7.7)$ & 5566 & $12.2(12.0-12.3)$ & 11,873 & $6.3(6.3-6.4)$ \\
\hline Cochlear implant & 2062 & $3.5(2.6-4.8)$ & 1708 & $3.7(2.8-5.0)$ & 4544 & $2.4(1.8-3.2)$ \\
\hline Congenital immunodeficiency & 4409 & $7.5(7.0-8.0)$ & 5919 & $12.9(12.6-13.3)$ & 14,738 & $7.9(7.5-8.2)$ \\
\hline Diseases of white blood cells & 6048 & $10.3(9.7-11.0)$ & 6797 & $14.8(14.6-15.1)$ & 13,262 & $7.1(6.9-7.3)$ \\
\hline Functional/anatomic asplenia & 8566 & $14.6(13.9-15.3)$ & 8721 & $19.0(18.7-19.4)$ & 15,976 & $8.5(8.3-8.7)$ \\
\hline HIV & 2291 & $3.9(2.8-5.5)$ & 2457 & $5.4(5.2-5.5)$ & 6461 & $3.4(3.2-3.8)$ \\
\hline Immuno. conditions/drugs & 3595 & $6.1(5.9-6.4)$ & 3146 & $6.9(6.8-6.9)$ & 7248 & $3.9(3.8-3.9)$ \\
\hline
\end{tabular}

of asthma (Additional file 1: Figure S1) and diabetes (Additional file 1: Figure S2).

\section{Discussion}

Our results, based on analyses of data from three large repositories of healthcare claims, suggest that the increased rates of pneumococcal disease associated with certain comorbid conditions (i.e., among at-risk and high-risk persons) are closely aligned with increased healthcare costs in these populations. Elevated rates and higher episodic costs of IPD and PNE in at-risk and high-risk populations resulted in total healthcare costs that were 3-43 times higher (per 100,000 personyears)-depending on age and condition-compared with age-stratified healthy counterparts. Among adults aged 18-64 years, for example, the annual cost of PNE (per 100,000 persons) increased from $\$ 1.8$ million for healthy persons, to $\$ 13.9$ million for at-risk persons, and to $\$ 50.4$ million for high-risk persons. Underlying the higher overall costs are increasing rates of disease (from 458 , to 1652 , to 3094 per 100,000 persons) and increasing mean cost per episode of disease (from \$4247, to
$\$ 8789$, to $\$ 16,775)$. The increase in mean cost per episode is especially pronounced for cases of disease requiring inpatient care $(\$ 18,224, \$ 21,815$, and $\$ 31,233$ across risk groups), which correlates with longer mean length of stay in hospital (12.8 days, 17.0 days, and 23.4 days, respectively). While a similar finding was expected for IPD, these results were not robust due to the relatively small number of identified episodes. Remarkably, while at-risk persons with $\geq 2$ conditions and highrisk persons accounted for only $8 \%$ of the population, they were responsible for more than $50 \%$ of total IPD/ PNE costs. In addition, among persons with at-risk conditions, elevated costs increased in a graded and monotonic fashion with the number of conditions present, and rates and costs of PNE were particularly high among persons with severe asthma and diabetes versus those with less severe disease.

The pattern of increasing costs across increasing levels of risk is similar to that observed in the abovecited prior study of the burden of pneumococcal disease among older US adults [2]. Although the risk categories considered in the two studies are similar, 
Table 2 Costs and resource use per episode of invasive pneumococcal disease and all-cause pneumonia among healthy, at risk, and high-risk persons

\begin{tabular}{|c|c|c|c|c|c|c|c|c|c|c|c|c|c|c|c|c|c|c|}
\hline & \multicolumn{9}{|c|}{ Invasive pneumococcal disease } & \multicolumn{9}{|c|}{ All-cause pneumonia } \\
\hline & \multicolumn{3}{|l|}{$<18$ years } & \multicolumn{3}{|c|}{$18-64$ years } & \multicolumn{3}{|c|}{$\geq 65$ years } & \multicolumn{3}{|l|}{$<18$ years } & \multicolumn{3}{|c|}{ 18-64 years } & \multicolumn{3}{|c|}{$\geq 65$ years } \\
\hline & INP & $\mathrm{OP}$ & Overall & INP & $\mathrm{OP}$ & Overall & INP & $\mathrm{OP}$ & Overall & INP & $\mathrm{OP}$ & Overall & INP & $\mathrm{OP}$ & Overall & INP & $\mathrm{OP}$ & Overall \\
\hline \multicolumn{19}{|l|}{ Risk Group } \\
\hline \multicolumn{19}{|l|}{ Healthy } \\
\hline \multicolumn{19}{|l|}{ Cost } \\
\hline Mean & $\$ 50,744$ & $\$ 448$ & $\$ 32,879$ & $\$ 51,695$ & $\$ 499$ & $\$ 43,349$ & $\$ 22,733$ & $\$ 605$ & $\$ 20,897$ & $\$ 12,130$ & $\$ 484$ & $\$ 3280$ & $\$ 18,224$ & $\$ 592$ & $\$ 4247$ & $\$ 12,587$ & $\$ 487$ & $\$ 7015$ \\
\hline SD & $\$ 82,620$ & $\$ 1540$ & $\$ 70,509$ & $\$ 84,974$ & $\$ 1148$ & $\$ 80,000$ & $\$ 28,405$ & $\$ 963$ & $\$ 27,876$ & $\$ 50,885$ & $\$ 717$ & $\$ 25,431$ & $\$ 37,336$ & $\$ 1043$ & $\$ 18,463$ & $\$ 26,113$ & $\$ 1141$ & $\$ 20,127$ \\
\hline LOS (mean) & 11.0 & - & - & 12.2 & - & - & 9.7 & - & - & 7.6 & - & - & 12.8 & - & - & 11.4 & - & - \\
\hline No. OP Visits (mean) & - & 1.1 & - & - & 1.8 & - & - & 2.8 & - & - & 1.2 & - & - & 1.4 & - & - & 1.8 & - \\
\hline $\mathrm{n}$ & 118 & 65 & 183 & 498 & 97 & 595 & 199 & 18 & 217 & 11,123 & 35,206 & 46,329 & 16,633 & 63,609 & 80,242 & 13,898 & 11,848 & 25,746 \\
\hline \multicolumn{19}{|l|}{ At-Risk } \\
\hline \multicolumn{19}{|l|}{ Cost } \\
\hline Mean & $\$ 101,491$ & $\$ 500$ & $\$ 47,111$ & $\$ 46,000$ & $\$ 1531$ & $\$ 39,377$ & $\$ 29,781$ & $\$ 412$ & $\$ 27,472$ & $\$ 19,207$ & $\$ 550$ & $\$ 7659$ & $\$ 21,815$ & $\$ 599$ & $\$ 8789$ & $\$ 13,719$ & $\$ 503$ & $\$ 8472$ \\
\hline SD & $\$ 147,671$ & $\$ 927$ & $\$ 110,596$ & $\$ 64,018$ & $\$ 4362$ & $\$ 61,160$ & $\$ 93,161$ & $\$ 662$ & $\$ 89,765$ & $\$ 46,187$ & $\$ 792$ & $\$ 29,922$ & $\$ 46,562$ & $\$ 1073$ & $\$ 30,729$ & $\$ 26,672$ & $\$ 1461$ & $\$ 21,716$ \\
\hline LOS (mean) & 17.3 & - & - & 11.1 & - & - & 10.7 & - & - & 12.7 & - & - & 17.0 & - & - & 14.8 & - & - \\
\hline No. OP Visits (mean) & - & 1.2 & - & - & 2.3 & - & - & 2.3 & - & - & 1.2 & - & - & 1.4 & - & - & 2.0 & - \\
\hline $\mathrm{n}$ & 12 & 14 & 26 & 360 & 63 & 423 & 457 & 39 & 496 & 4007 & 6508 & 10,515 & 18,583 & 29,554 & 48,137 & 36,714 & 24,178 & 60,892 \\
\hline \multicolumn{19}{|l|}{ High-Risk } \\
\hline \multicolumn{19}{|l|}{ Cost } \\
\hline Mean & $\$ 29,896$ & $\$ 128$ & $\$ 22,891$ & $\$ 45,406$ & $\$ 715$ & $\$ 42,985$ & $\$ 23,809$ & $\$ 1714$ & $\$ 21,674$ & $\$ 35,721$ & $\$ 612$ & $\$ 20,304$ & $\$ 31,233$ & $\$ 750$ & $\$ 16,775$ & $\$ 15,090$ & $\$ 571$ & $\$ 9495$ \\
\hline SD & $\$ 23,936$ & $\$ 83$ & $\$ 24,476$ & $\$ 52,315$ & $\$ 656$ & $\$ 51,872$ & $\$ 30,280$ & $\$ 4196$ & $\$ 29,536$ & $\$ 101,049$ & $\$ 925$ & $\$ 77,632$ & $\$ 62,950$ & $\$ 2477$ & $\$ 48,142$ & $\$ 26,296$ & $\$ 1745$ & $\$ 21,820$ \\
\hline LOS (mean) & 8.6 & - & - & 12.5 & - & - & 9.8 & - & - & 18.5 & - & - & 23.4 & - & - & 16.7 & - & - \\
\hline No. OP Visits (mean) & - & 1.0 & - & - & 3.0 & - & - & 4.0 & - & - & 1.3 & - & - & 1.6 & - & - & 2.3 & - \\
\hline $\mathrm{n}$ & 13 & 4 & 17 & 227 & 13 & 240 & 271 & 29 & 300 & 525 & 411 & 936 & 8480 & 7651 & 16,131 & 17,809 & 11,165 & 28,974 \\
\hline
\end{tabular}

INP inpatient, OP outpatient 


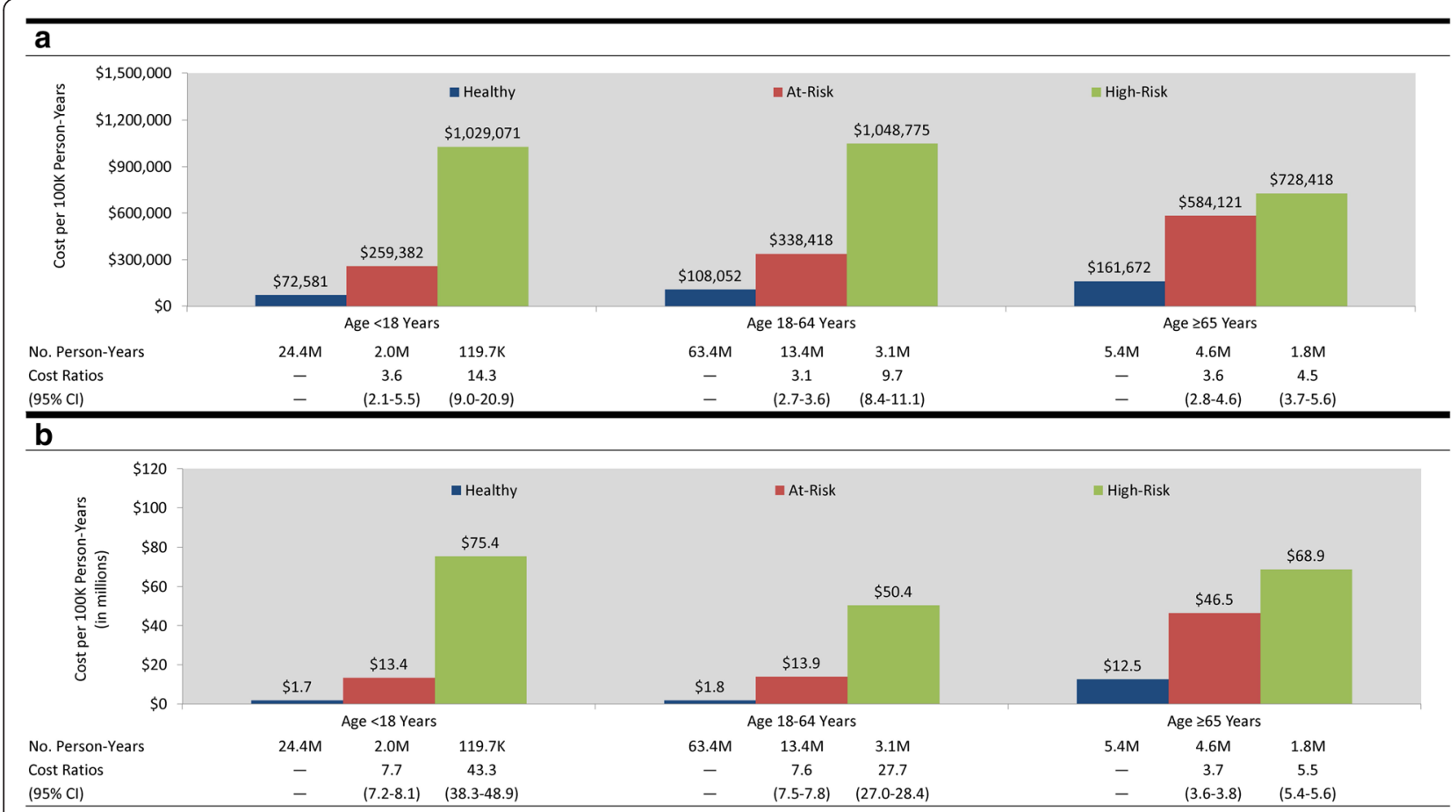

Fig. 1 a Annual cost of invasive pneumococcal disease per 100,000 healthy, at-risk, and high-risk persons. b Annual cost of all-cause pneumonia per 100,000 healthy, at-risk, and high-risk persons

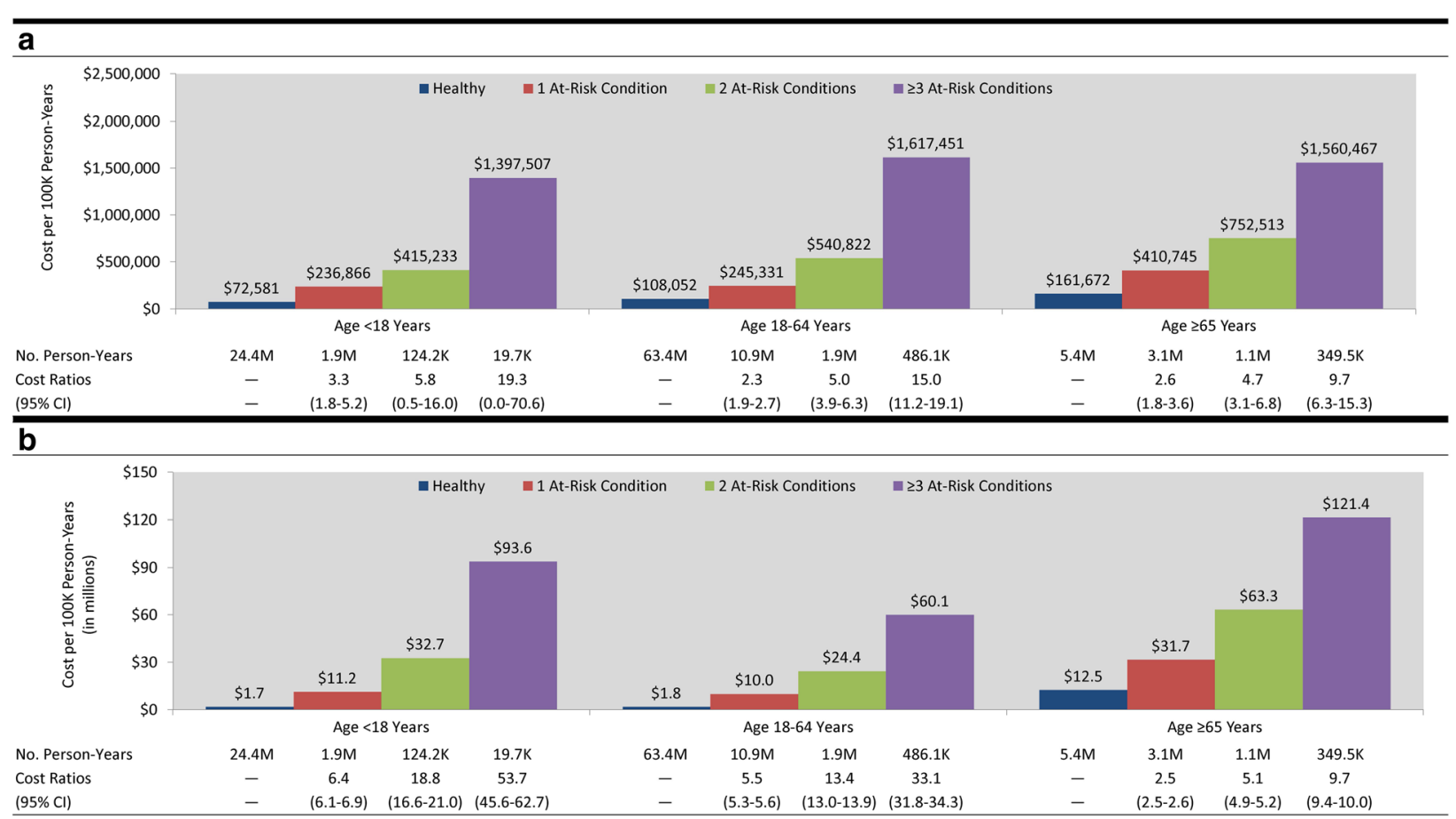

Fig. 2 a Annual cost of invasive pneumococcal disease per 100,000 persons with at-risk conditions - by number of conditions - versus their healthy counterparts. b Annual cost of all-cause pneumonia per 100,000 persons with at-risk condition - by number of conditions - versus their healthy counterparts 


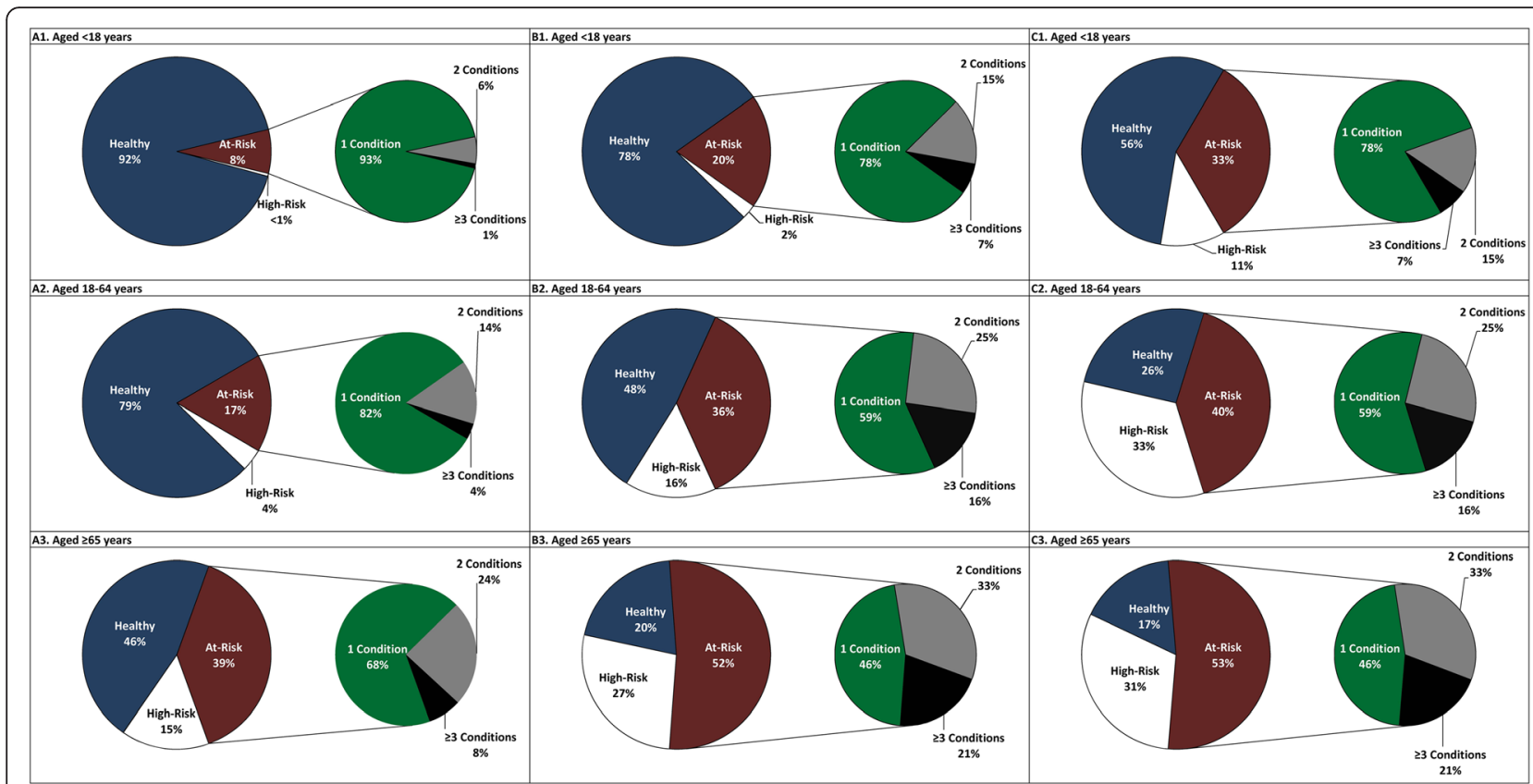

Fig. 3 Distribution of persons by risk profile and among at-risk persons by number of conditions (Panel a), distribution of pneumococcal disease episodes among at-risk persons by number of conditions (Panel $\mathbf{b}$ ), and distribution of pneumococcal disease costs among persons at-risk by number of conditions (Panel $\mathbf{c}$ )

direct comparisons of costs with the prior study are challenging because they were estimated from different sources and were presented in a different manner. In the prior study, for example, direct costs of inpatient care were estimated on the basis of reported charges for the relevant conditions in the 2004 Healthcare Cost and Utilization Project Nationwide Inpatient Sample (HCUP NIS), after "stepping-down" costs to charges using hospital-specific cost-to charge ratios. In the current study, inpatient costs were estimated from paid amounts available in healthcare claims. In the prior study, costs were presented for the entire US population of older adults (i.e., those aged $\geq 50$ years) stratified into four age groups, while in the current study costs were presented per 100,000 adults aged 19-64 years and $\geq 65$ years, respectively.

Two additional findings merit further discussion. First, rate and cost ratios for IPD and PNE were similar. This finding likely is explained by two factors. The predominant manifestation of IPD is bacteremic pneumonia-69\% of all cases in the US in 2012-and pneumococcus is believed to be the leading bacterial cause of all-cause pneumonia $[8,10]$. Thus, to a considerable extent, these two outcomes overlap and represent the same clinical manifestation-pneumococcal pneumonia. In addition, conditions that predispose to pneumonia caused by $S$. pneumoniae may increase the risk of disease due to other pathogens. For example, chronic lung disease probably increases the risk of pneumonia caused by influenza virus. The second finding was that rate ratios were smaller in older persons. We believe this finding to be a manifestation of the general decrease in immunocompetence that occurs with advancing age and that almost certainly diminishes the risk gradient between "healthy" older adults and those with established chronic medical conditions.

While healthcare claims databases provide information on large numbers of patients with specific diagnoses and the associated healthcare utilization, several limitations from the use of such databases for our study should be noted. First, use of operational algorithms and healthcare claims data resulted in an underestimation of the incidence of IPD (e.g., $\sim 8$ per 100,000 children $<5$ years of age) compared with national estimates from the Centers for Disease Control and Prevention (e.g., 20 per 100,000 children $<5$ years of age) [10]. For IPD, it is likely that sometimes only the ICD-9-CM diagnosis code for unspecified septicemia or bacteremia is recorded on the healthcare claim form, without an accompanying diagnosis code for pneumococcus. Thus, while such encounters would be correctly identified as invasive disease, they would not be correctly identified as IPD. In addition, private healthcare claims databases-like the ones used in this study-include information for commercially insured populations, which often have an overall lower risk of disease. We note, however, that the age distribution of IPD cases in our study is similar to the distribution that has been reported by the Centers for 
Disease Control and Prevention (CDC). While it is not possible to formally evaluate the accuracy of our caseascertainment algorithms within the context of this study, we did evaluate the sensitivity of our study results by employing alternative approaches to characterizing risk profiles and individual conditions. In one approach, relevant data available during the calendar year were considered as evidence that such conditions were present at the beginning of the calendar year, while in another approach, such evidence was treated as a change in risk status during the calendar year. Analyses also were conducted using less restrictive (i.e., more sensitive) algorithms in which any diagnostic, procedure, or drug information was considered evidence that such conditions were present. In addition, analyses were conducted by individual calendar year, with varying durations of look-back to ascertain risk profiles (e.g., $\leq 1$ year for 2007, $\leq 4$ years for 2010). Across all of the abovedescribed analyses, our findings were largely unchanged.

Second, persons with public or no health insurance are not represented in the study databases, potentially limiting the generalizability of study results to other populations. Third, the costs of PNE are not a substitute for the costs of pneumococcal pneumonia, and thus our study undoubtedly overestimated the absolute clinical and economic burden of pneumococcal disease. For this reason, our study focused on rate ratios and cost ratios, rather the rates and costs per se. Assuming that the costs of pneumococcal pneumonia are similar to the costs of pneumonia due to infection with other pathogens, the costs of pneumococcal pneumonia would be proportional to the percentage of all-cause pneumonia due to $S$. pneumoniae, recently estimated for community-acquired pneumonia at $27 \%$ (95\%CI 21-29) [8]. Fourth, this study employed a retrospective cohort design and data from three large integrated healthcare claims databases to increase the precision of estimates. Although the three study databases comprise information from unique health plans and thus do not overlap at the plan level, it is possible that claims information from different time periods for the same patient may appear in each extract (and cannot be linked because of unique encrypted IDs). We believe such occurrences to be rare and the potential bias-if any-to be minimal. Finally, we did not include data on pneumococcal vaccination status in our analyses due to the lack of reliable information on this subject.

Several generic limitations of retrospective studies based on healthcare claims data also should be noted. All healthcare claims databases contain errors of omission and commission in coding, which undoubtedly results in some misclassification of study outcomes. Certain biases in coding may exist such that patients who, for example, are hospitalized for IPD or PNE may be more likely to have selected underlying at-risk/high-risk conditions listed as primary/secondary diagnoses on their claims, all else equal. Moreover, information often is not available for one or more clinically important parameters (in our study, for example, blood glucose/ glycated hemoglobin [HbA1c] for diabetes, ejection fraction/New York Heart Association [NYHA] Class for heart failure), and pertinent medical history can be leftcensored (e.g., diagnoses recorded before the time period of the study database are unobservable). The impact of these limitations on our results cannot be assessed within the scope of this study.

\section{Conclusion}

In conclusion, the results of this study demonstrate that rates and costs of pneumococcal disease remain disproportionately high in persons with at-risk and high-risk conditions in the current era of widespread pneumococcal vaccination. Study results also demonstrate that rates and costs for individuals with $\geq 2$ at-risk conditions approached those among persons with high-risk conditions, and that the rates and costs for persons with asthma and diabetes were especially increased.

\section{Ethics}

Because the study databases were de-identified prior to their release to study investigators, their use for health services research is fully compliant with the HIPAA Privacy Rule and US federal guidance on Public Welfare and the Protection of Human Subjects. Formal IRB approval was therefore not required.

\section{Availability of data and materials}

Use of the data for analyses described herein is restricted via license agreements between study investigators and the data vendors, and thus the data are not publically available. Use of the data sources for future research may be obtained via user-specific license agreements with the data vendors.

\section{Additional file}

Additional file 1: Supplemental Material-Operational Algorithms and

Additional Results. (DOCX 2446 kb)

\section{Abbreviations \\ ACIP: Advisory Committee on Immunization Practices; CDC: Centers for Disease Control and Prevention; HbA1c: glycated hemoglobin; HCPCS: Healthcare Common Procedure Coding System; HCUP NIS: Healthcare Cost and Utilization \\ Project Nationwide Inpatient Sample; HIPAA: Health Insurance Portability and Accountability Act; ICD-9-CM: International Classification of Diseases, Ninth Revision, Clinical Modification; IMS: Intercontinental Marketing Services'; IPD: invasive pneumococcal disease; IRR: incidence rate ratio; NDC: National Drug Code; NYHA: New York Heart Association; PNE: pneumonia; US: United States.}

Competing interests

Derek Weycker and John Edelsberg are employees of PAI, which received

financial support from Pfizer Inc. for this study (including manuscript preparation). 
Raymond Farkouh and David Strutton are employed by, and own stock in, Pfizer Inc. Kimberly M. Shea, employed by Boston University Schools of Medicine and Public Health, and Stephen I. Pelton, employed by Boston University Schools of Medicine and Public Health and Boston Medical Center, received financial support from Pfizer Inc. for their participation in study design, data analysis, and data interpretation. Kimberly M. Shea has received an investigator-initiated research grant from Pfizer Inc. Stephen I. Pelton has served as an advisory board member for, and received investigator-initiated research grants from, Pfizer Inc. and other vaccine manufacturers.

\section{Authors' contributions}

Authorship followed the guidelines proposed by the International Committee of Medical Journal Editors (2004). All authors meet the criteria for authorship, and all people who meet the criteria are authors. Potential conflicts of interest have been disclosed. All authors have: (1) made substantial contributions to conception and design (JE, RAF, SIP, KMS, DRS, DW), acquisition of data (RAF, DRS, DW), or analysis and interpretation of data (JE, RAF, SIP, KMS, DRS, DW); (2) participated in drafting the article or revising it critically for important intellectual content (JE, RAF, SIP, KMS, DRS, DW); and (3) approved the version to be published (JE, RAF, SIP, KMS, DRS, DW). The study sponsor, Pfizer Inc., reviewed the study research plan and study manuscript; data management, processing, and analyses were conducted by PAl, and all final analytic decisions were made by study investigators. DW confirms that he had full access to all the data in the study and had final responsibility for the decision to submit for publication.

\section{Acknowledgements}

We thank Rebecca Bornheimer and Aaron Moynahan of Policy Analysis Inc. (PAI) for their assistance with data analysis.

\section{Source of funding}

Funding for this research was provided by Pfizer Inc. to Policy Analysis Inc. (PAI).

\section{Author details}

'Policy Analysis Inc. (PAI), Four Davis Court, Brookline, MA 02445, USA. ${ }^{2}$ Pfizer Inc., Collegeville, PA, USA. ${ }^{3}$ Boston University Schools of Medicine and Public Health, Boston, MA, USA. ${ }^{4}$ Boston Medical Center, Boston, MA, USA.

Received: 26 November 2014 Accepted: 10 May 2016

\section{Published online: 13 May 2016}

\section{References}

1. Huang SS, Johnson KM, Ray GT, et al. Healthcare utilization and cost of pneumococcal disease in the United States. Vaccine. 2011;29(18):3398-412.

2. Weycker D, Strutton D, Edelsberg J, et al. Clinical and economic burden of pneumococcal disease in older US adults. Vaccine. 2010;28:4955-60.

3. Wroe PC, Finkelstein JA, Ray GT, et al. Aging Population and Future Burden of Pneumococcal Pneumonia in the United States. J Infect Dis. 2012;205(10):1589-92.

4. Wotton CJ, Goldacre MJ. Risk of invasive pneumococcal disease in people admitted to hospital with selected immune-mediated diseases: record linkage cohort analyses. J Epidemiol Community Health. 2012;66(12):1177-81.

5. Vinogradova Y, Hippisley-Cox J, Coupland C. Identification of new risk factors for pneumonia: population-based case-control study. Br J Gen Pract. 2009;59(567):e329-38.

6. Bhat N, Wright JG, Broder KR, et al. Influenza-associated deaths among children in the United States, 2003-2004. N Engl J Med. 2005;353(24):2559-67.

7. CDC. Use of 13-Valent Pneumococcal Conjugate Vaccine and 23-Valent Pneumococcal Polysaccharide Vaccine for Adults with Immunocompromising Conditions: Recommendations of the Advisory Committee on Immunization Practices (ACIP). MMWR Morb Mortal Wkly Rep. 2012;61:816-9.

8. Said MA, Johnson HL, Nonyane BA, et al. Estimating the burden of pneumococcal pneumonia among adults: a systematic review and meta-analysis of diagnostic techniques. PLoS ONE. 2013;8(4):e60273. doi:10.1371/journal.pone.0060273.

9. US Department of Health and Human Services: Code of Federal Regulations: Title 45, public welfare; Part 46, protection of human subjects. Effective July 14, 2009. Available: [http://www.hhs.gov/ohrp/humansubjects/guidance/ 45cfr46.html]. Accessed 4 July 2013

10. CDC: Active Bacterial Core Surveillance report, Emerging Infections Program Network, Streptococcus pneumoniae, 2012. Available: [http://www.cdc.gov/ abcs/reports-findings/survreports/spneu12.html]. Accessed 4 July 2013.

\section{Submit your next manuscript to BioMed Central and we will help you at every step:}

- We accept pre-submission inquiries

- Our selector tool helps you to find the most relevant journal

- We provide round the clock customer support

- Convenient online submission

- Thorough peer review

- Inclusion in PubMed and all major indexing services

- Maximum visibility for your research

Submit your manuscript at www.biomedcentral.com/submit 